\title{
LA AUTORREGULACIÓN: UN AVANCE HACIA LA AUTONOMÍA DE ENFERMERÍA ${ }^{1}$
}

\author{
SELF-REGULATION: A STEP TOWARDS \\ NURSING AUTONOMY
}

\section{AUTORREGULAÇÃO: UM AVANÇO EM DIREÇÃO À AUTONOMIA DA ENFERMAGEM}

\author{
Luz Galdames Cabrera* \\ Bertha Cruz Enders** \\ Amaya Pavez Lizarraga***
}

\begin{abstract}
RESUMEN
La profesión de Enfermería en Chile se vio afectada por los hechos ocurridos en la sociedad chilena desde la Constitución de 1980 y la modificación del Código Sanitario en 1981, a través del Decreto Ley 3621. La construcción de la definición de Enfermería introducida al Código Sanitario chileno en el año 1997 proporcionó el respaldo legal a la función social de la enfermera, reconociendo a su vez a Enfermería como una profesión autónoma. Objetivo: Analizar cómo se gestó la construcción de la definición de Enfermería que se introdujo en el Código Sanitario chileno, en su artículo No 113. Método: Estudio de abordaje socio-histórico, a través del testimonio de dos enfermeras que fueron actores relevantes en la construcción de la definición de Enfermería. Las entrevistas fueron analizadas a través del análisis de discurso. Resultados: Se expone una reflexión crítica realizada por enfermeras visionarias para lograr obtener una definición de Enfermería, que reconoce legalmente a la profesión en su función social, principalmente en el cuidado de la salud de las personas. Conclusión: Este hecho histórico que legitimó la función social de la enfermera significó un proceso reivindicativo al mandato social de la labor de la enfermera y constituyó un hito sociopolítico, sin embargo, a más de veinte años aparecen nuevos desafíos en el área de la salud y cuidado de las personas, cobrando valor continuar con la autorregulación para consolidar su autonomía e identidad profesional.
\end{abstract}

Palabras clave: Enfermería; Regulación; Autonomía profesional; Identificación social.

\footnotetext{
${ }^{1}$ Extraído de Tesis de Doctorado realizada en el Programa de Doctorado en Enfermería de la Universidad Andrés Bello, Chile.

* Enfermera. Escuela de Enfermería, Facultad de Ciencias, Universidad Mayor, Chile. Email: luz.galdames@umayor.cl. ** Enfermera. Profesora Departamento de Enfermagen, Universidad Federal do Rio Grande do Norte, Brasil. Email: berthacruz.enders@gmail.com

*** Enfermera-matrona, antropóloga social. Profesora Salud Pública Facultad de Ciencias Médicas de la Universidad de Santiago, Chile. Email: amaya.pavez@usach.cl.
} 


\begin{abstract}
The nursing profession in Chile was affected by events that occurred in the Chilean society since the Constitution of 1980 and the modification of the Health Code in 1981, through the DL 3621 (Executive order). The construction of the definition of nursing introduced to the Chilean Health Code in the year 1997 provided legal support to the social function of the nurse, recognizing nursing as an autonomous profession. Objective: To analyze how the construction of the definition of nursing that was introduced in the Chilean Health Code, in its article No. 113, was developed. Method: Socio-historical approach, through the testimony of two nurses who were relevant actors in the construction of the definition of nursing. The interviews were analyzed through discourse analysis. Results: We present a critical reflection made by visionary nurses to construct a definition of nursing, which legally recognizes the profession in its social function, mainly in people's health care. Conclusion: The historical event that legitimized the social function of the nurse meant a vindicating process to the social mission of the nurse's work and became a sociopolitical milestone, however, more than twenty years later new challenges arise in the area of people's health care, giving new value to selfregulation to strengthen autonomy and professional identity.
\end{abstract}

Key words: Nursing; Regulation; Professional Autonomy; Social Identification.

\title{
RESUMO
}

A profissão de enfermagem no Chile foi afetada pelos acontecimentos na sociedade chilena desde a Constituição de 1980 e a modificação do Código Sanitário em 1981, através do DL 3621. A construção da definição de Enfermagem inserida no Código Sanitário chileno o ano de 1997 forneceu o suporte legal à função social da enfermeira, reconhecendo a Enfermagem como uma profissão autônoma. Objetivo: Analisar como foi construída a definição de enfermagem que foi inserida no Código Sanitário chileno, no artigo no 113. Método: Estudo de abordagem sócio-histórica, através do depoimento de duas enfermeiras que foram atores relevantes na construção da definição de enfermagem. As entrevistas foram analisadas por meio da análise do discurso. Resultados: Apresenta-se uma reflexão crítica feita por enfermeiras visionárias para obter uma definição de Enfermagem, que legalmente reconhece a profissão em sua função social, principalmente no cuidado da saúde das pessoas. Conclusão: Este fato histórico que legitimou a função social da enfermaria significou um processo reivindicatório á missão social do trabalho do enfermeiro e significou um marco sócio-político, porém, mais de vinte anos depois existem novos desafios na saúde e no cuidado das pessoas, ganhando valor continuar com a auto-regulação para consolidar sua autonomia e identidade profissional.

Palavras chave: Enfermagem; Regulação; Autonomia Profissional; Identificação Social.

Fecha recepción: 27/04/2018 Fecha aceptación: 02/05/2019

\section{INTRODUCCIÓN}

La Enfermería desde sus inicios como profesión, ha estado en la búsqueda de su identidad y la definición de sus dominios en el campo de la salud y el cuidado de las personas ${ }^{(1)}$. La identidad y los dominios se reflejan en la posesión de un cuerpo de conocimiento propio, un código de ética, marcos regulatorios y una autonomía profesional que permiten se la distinga de otras profesiones ${ }^{(1,2)}$. "La regulación profesional define la profesión y a sus miembros, determina el alcance de la práctica, establece los estándares de educación, una práctica ética y competente, y los sistemas de responsabilidad"(3). A 
su vez la autorregulación profesional es considerada un privilegio, ya que acentúa el poder político, social y económico de los profesionales involucrados ${ }^{(4)}$.

Desde la perspectiva del reconocimiento social, algunos autores reseñan las características que se relacionan con la definición de la profesión: la posesión de un cuerpo único de conocimiento, la provisión de un servicio altruista a la sociedad, la autonomía en el sentido de control sobre su trabajo y las condiciones laborales que reflejan parcialmente la realidad del trabajo en salud ${ }^{(1,2,5)}$.

Desde una perspectiva histórica y social, la Enfermería en Chile se ha desarrollado, como en otras realidades, siguiendo los periodos vocacional, técnico y profesional ${ }^{(6)}$. Estos períodos ocurren secuencialmente, pero a la vez se traslapan uno con otro. La etapa vocacional de la historia chilena está marcada por la fuerte influencia religiosa que siguió a la conquista española y la Colonia hasta avanzada la República. Luego, la etapa técnica destaca por el desarrollo de la Enfermería con una fuerte connotación en lo biomédico, enraizado aún en lo religioso. La formación hace énfasis en lo curativo e integra lo preventivo con la salud comunitaria; la función de la enfermera sanitaria fue clave en la mejora de las condiciones de salud de la población. El período profesional se caracteriza por el movimiento nacional de las enfermeras para establecerse como una profesión en el país, donde buscaron empoderarse a través del Colegio Profesional ${ }^{(6)}$. A la década de los setenta en Chile se la reconoce marcada por los cambios y confrontaciones en lo político y social, que ocurrieron como consecuencia del régimen militar imperante de la época ${ }^{(6)}$. Debido a los cambios sociales que se produjeron y la instauración de la Constitución de $1980^{(7)}$, las enfermeras perdieron el espacio profesional cuando se descentralizó la estructura del Sistema Nacional de Salud (SNS), quedando sin un respaldo en la estructura orgánica y jerárquica de los servicios de salud. De esta forma desaparecieron los servicios de enfermería en los hospitales al instalarse la supremacía de la autoridad médica en las instituciones de salud, limitando la toma de decisiones de la enfermera, en cuanto al cuidado de la salud de las personas y la tuición sobre el personal de enfermería.

Estas acciones tuvieron consecuencia en la autonomía profesional que las enfermeras habían logrado consuetudinariamente en las instituciones de salud. Posteriormente la modificación del Código
Sanitario publicado en 1981, DL $3621^{(7)}$, suprime los colegios profesionales como corporaciones de derecho público, pasando a ser Asociaciones Gremiales (A.G.), por lo cual los profesionales no tienen obligación de pertenecer a estas asociaciones y los colegios perdieron el control ético sobre los colegiados y su facultad de imperio ${ }^{(7)}$. Es así como en el año 1982 el Colegio de Enfermeras se transforma de institución de derecho público en institución de derecho privado, denominándose desde ese año como Colegio de Enfermeras de Chile A.G. ${ }^{(6)}$. Esto tiene como consecuencia que los estatutos o reglamentos que instruían sobre la función social de la enfermera hayan sido anulados, llevando a una desregulación de la profesión, y de su mandato social. Por otro lado, el ejercicio libre de la profesión tuvo como consecuencia la pérdida de la fuerza política de la asociación gremial, transformándose en una agrupación que puede velar por el ejercicio de la profesión, pero su opinión no es vinculante para renovar y revitalizar el mandato social de la profesión, como declara el Consejo Internacional de Enfermeras (CIE) en cuanto a la regulación de las enfermeras $^{(6,7)}$.

Posteriormente, en el año 1997 se introdujo al Código Sanitario chileno en su artículo No $113^{(8)}$ una nueva definición de enfermería, que proporcionó el respaldo legal a la función social de la enfermera en el país, reconociendo a su vez a Enfermería como una profesión autónoma, siguiendo la tendencia internacional ${ }^{(9)}$. Este artículo tiene como objetivo analizar cómo se gestó la construcción de la definición de Enfermería que se introdujo en el Código Sanitario chileno de 1997, en su artículo No 113.

\section{MATERIAL Y MÉTODO}

Este artículo se basa en una investigación cualitativa socio-histórica más amplia titulada Gestión del cuidado: comprensión de los significados del rol social y autonomía profesional de la enfermera en Chile.

Se realizaron entrevistas a informantes clave, para ello fueron invitadas a participar dos enfermeras que se constituyeron en actores relevantes en la construcción de la definición de enfermería. Fueron consideradas informantes clave por estar ubicadas, en ese momento, en espacios estratégicos, una de ellas en el Ministerio de Salud y la otra, enfermera- 
abogada, precursora de la Comisión de legislación formada el año 1992.

Ambas informantes fueron contactadas telefónicamente, solicitando su disposición a relatar en dos entrevistas su participación en este proceso sociopolítico. Se obtuvo su consentimiento informado y se resguardó su anonimato mediante la codificación de su identidad. Las entrevistas tuvieron una duración promedio de dos horas, siendo transcritas en su totalidad para su lectura exhaustiva antes de realizar la siguiente entrevista. En la segunda entrevista se validó por las informantes los antecedentes de los hechos relatados, lo que permitió un análisis secuencial y continuo. Posteriormente, los fragmentos de los discursos se agruparon en la secuencia lógica de los hechos acaecidos en la época.

La información obtenida fue analizada desde el análisis de discurso ${ }^{(10)}$. La investigación obtuvo la aprobación del Comité de Ética en Investigación de la Facultad de Enfermería de la Universidad Andrés Bello, Folio L1/CECENF/95.

\section{RESULTADOS}

A continuación se presenta segmentos de los testimonios de los informantes claves que dan cuenta, primero, de cómo el cambio de modelo de sociedad que se instauró en el país con la Constitución de 1980 y la modificación del Código Sanitario en 1981, a través del DL 3621 que suprimió los colegios profesionales, repercutió en el ejercicio profesional de las enfermeras y, en segundo lugar, cómo las enfermeras líderes de ese período lograron transformar este efecto negativo en una oportunidad de empoderamiento al abrir el espacio para reivindicar el derecho a ejercer el cuidado de la salud. Es decir, apropiarse legalmente del objeto de la profesión enraizado en su ontología de cuidado de la salud.

\section{(...) La legitimación social que las enfermeras} teniamos no era suficiente. El Colegio de Enfermeras en su estatuto definía las funciones de la enfermera, este estatuto, no era universal y pasa a ser insuficiente al existir la libertad de colegiarse para ejercer la profesión, lo que obliga a las enfermeras a definir su función social, acción que requeria precisar los problemas sociales que resuelve la enfermera, y no hay discusión, de que la enfermera resuelve los problemas relacionados al cuidado (EIC 2).

Esto originó que, a partir de 1981, se produjera una reacción de las enfermeras para la defensa legal de la profesión, promovida por actores sociales que lograron hacer una reflexión crítica y prever los alcances que tendría la pérdida de la tuición de sus asociados, permitiéndose el ejercicio libre de la profesión.

\section{(...) En el año 1992 formamos la Comisión de} legislación, la constituimos por representantes de las Sociedades Cientificas, Asociación Chilena de Educación en Enfermería y el Colegio Profesional (EIC 2).

La reivindicación de las demandas y argumentos alcanzaron su punto de inflexión en el año 1992, cuando se constituyeron en referentes para formar la Comisión de Legislación.

Por otra parte, a lo anterior se suman los hechos que ocurrían en paralelo en organismos internacionales como en la Organización Panamericana de la Salud (OPS), que en el año 1994 en su 54a Asamblea identifica la necesidad de legislar en Enfermería al interior de los países de la región.

(...) Estando como Asesora en el Ministerio de Salud, recibi desde la Organización Panamericana de la Salud OPS el acuerdo de la Asamblea No 54 del año 1994, de apoyar a los paises de América Latina en la legislación de Enfermería, se debia preparar un proyecto en menos de una semana y llamé a una de las personas que formaba parte de esta Comisión de Legislación para ver que podiamos hacer (EIC 1).

(...) Elaboré el proyecto sobre la marcha, lo denominé "Bases Legales para la Enfermería Chilena", BLECH, pasado un tiempo... me parece que fueron semanas no recuerdo bien..., recibimos la aprobación del proyecto con un presupuesto de 20.000 dólares (EIC 2).

(...) Recibida la aprobación, nos acercamos al Colegio de Enfermeras para dar a conocer el proyecto, esto no fue fácil, para la Asesora Ministerial, limar las asperezas fue complejo, la premura en presentar el proyecto nos hizo caer en la imprudencia de no sumar a otros, pero habia que aprovechar la oportunidad (EIC 2). 
El deber ser en enfermeras, con una impronta identitaria arraigada en la esencia de la Enfermería, se refleja en un compromiso con la profesión que conlleva acciones políticas en el contexto del colectivo, al trabajar articuladamente las fortalezas individuales con las gremiales, lo que rompe con la lógica que el nuevo modelo ideológico intentaba instaurar.

\section{(...) Durante la definición se debió buscar qué} diferenciaba al cuidado de enfermería del cuidado de otras profesiones, el hecho que la enfermera considera al ser humano en su totalidad y cuida a la persona en todo su ciclo vital desde el recién nacido hasta la adultez mayor, ya hacia una diferencia con otras profesiones. Además, la enfermera está en el planeamiento de ese cuidado, su formación reconocida en administración que le permite hacer el mejor uso de los recursos disponibles conforme a las necesidades del paciente (EIC 2).

La comisión ve una oportunidad para argumentar y fundamentar su postura de la contingencia regional entorno a la situación de salud y necesidades de profesionales de Enfermería, lo que fue una estrategia eficaz al poner el tema del cuidado y su inherencia a Enfermería en el contexto internacional y sacarlo del ámbito local donde los intereses particulares estaban en contra de la defensa de lo propio de la profesión.

\section{(...) El año 1995 se recibe de OPS la indicación} de congelar los apoyos económicos al extranjero. Como consecuencia de la crisis económica. Sin embargo, era de interés continuar apoyando el proyecto BLENCH de Chile, mediante la asesoría de dos consultores, con las cuáles se logró: i) definir los elementos que debia contener la legislación en enfermería (fuera ésta un estatuto, una norma o reglamento) y ii) elaborar el contenido para una norma jurídica que definiera enfermería y regulara el ejercicio profesional (EIC1).

(...) El trabajo con los consultores resultó ser crucial en la construcción de la definición legal de enfermería, la que se concluyó el año 1996, quedó archivada por más de un año a la espera de la instancia que permitiera ser presentada al Poder Legislativo (EIC1).

De la misma forma, la decisión de OPS del año 1996 de congelar el presupuesto asignado al proyecto $\mathrm{BLECH}$, lo que en un principio fue percibido como una amenaza por la Comisión Nacional de Legislación sucesora de la Comisión de Legislación, se transforma finalmente en una oportunidad para la consecución del proyecto, a través de la asesoría de dos expertas en regulación y legislación, concluyéndose la definición del rol y funciones de la enfermera en Chile.

\section{(...) Recién en el año 1997, mediante una ley} miscelánea, se generó la oportunidad para que la definición de enfermería se introdujera al Código Sanitario en octubre del mismo año (EIC1).

\section{DISCUSIÓN}

Los marcos regulatorios internacionales como los nacionales se constituyen en conceptualizaciones que definen los espacios de accionar de la enfermera, precisando el qué, el cómo y el porqué de Enfermería, en el campo de la salud. Ya en democracia en el año 1997 acontece un hito histórico para la profesión, se obtuvo el reconocimiento legal de la autonomía del ejercicio profesional ${ }^{(8)}$, que limitó el efecto de la ley anterior DL 3621. Si se observan los periodos de estas medidas, una limitante y otra progresiva como el reconocimiento legal de la autonomía profesional, se puede decir que las transformaciones políticas y económicas relacionadas con el sector salud han afectado el ámbito de la enfermería profesional, produciendo efectos negativos o positivos, con las consiguientes contradicciones observadas y evidenciadas en los diferentes procesos de transformación de nuestra sociedad.

Los cambios limitantes tuvieron como consecuencia la ruptura de la cohesión del colectivo, dando paso al surgimiento de la individualidad como protagonista social; es así que cada enfermera se reinventó así misma y a la profesión, dependiendo de las capacidades personales y las posibilidades que tuviesen, fragmentándose la identidad de la profesión. Esto tuvo como resultado la pérdida de la fuerza gremial, la profesión quedó a la deriva de las iniciativas individuales, sometida a la hegemonía médica que se instaló con fuerza en las instituciones desalud y del respaldo legal a través de la preeminencia de una profesión de salud sobre otras, por ser estas últimas definidas como colaboradoras ${ }^{(8)}$.

Sin embargo, es importante señalar que, a pesar de la abolición de los derechos sociales de la 
Enfermería como profesión que se produjeron como consecuencia de las reformas del Estado que siguieron a la Constitución de 1980, las enfermeras líderes de la época fueron capaces de hacer una reflexión crítica y mirar la realidad con otro lente, como señalan Chinn y Kramer ${ }^{(11)}$, en un proceso emancipatorio que duró más de una década. Mediante ese proceso se reorientó la visión tradicional sobre la esencia del ser y del hacer de la enfermera, visión emancipatoria que es referida en el desarrollo del conocimiento de Enfermería ${ }^{(12)}$.

La incorporación de la Enfermería chilena como profesión en el artículo 113 del Código Sanitario, se constituye en la primera instancia nacional, donde desde el Estado se visibiliza el cuidado como una necesidad, siendo, por lo tanto, el primer reconocimiento público al cuidado como una acción consustancial a la existencia humana. De tal forma, se puede decir que la definición de Enfermería y su incorporación en el Código Sanitario se produjo por una suerte de oportunidades que se dieron en el contexto sociopolítico a nivel nacional e internacional, y que las enfermeras líderes de la época, insertas en espacios de poder de ese período histórico supieron aprovechar, producto de su formación, su conciencia social, y de la importancia que tenía para ellas la profesión como colectivo transformador y promotor de la salud en el país ${ }^{(13-15)}$.

En la práctica, el significado que cobra el respaldo legal a la función social de la enfermera, y la autonomía profesional, proporcionan la oportunidad para que la profesión se diferencie de otros campos profesionales en cuanto a sus funciones ${ }^{(14,16,17)}$. Este fortalecimiento organizativo y político respecto al ejercicio liberal de la profesión, la introducción al mercado laboral de sus servicios expertos, la construcción de una identidad, el gobierno y la legislación fueron una expresión de su autonomía. Todo esto se percibe como un progreso para la profesión de Enfermería ${ }^{(18-20)}$.

La identidad de la enfermera comienza reconociéndose a sí misma en su ser enfermera y quehacer, diferenciándose de otros profesionales de la salud, y que a su vez sea reconocida en coherencia, es decir, que se produzca una armonía entre la autopercepción y la percepción social. Es así que cobra valor la autorregulación como un elemento importante de autonomía y consolidación de identidad; esto permite defender con mayor convicción la dimensión que diferencia Enfermería de otras profesiones, como lo es la definición de
Enfermería en el Código Sanitario.

Estos tres elementos: autorregulación, autonomía e identidad, forman un circulo virtuoso debido a su interdependencia, en que uno favorece la consolidación del otro, en un proceso sinérgico como señalan algunos autores ${ }^{(18,20,21)}$. Sin duda, el respaldo legal a la función de la enfermera es un avance para la profesión y constituye un hito político importante para el mandato social de la Enfermería como profesión en el país y en Latinoamérica. Desde una perspectiva histórica, el desarrollo conceptual y los progresos alcanzados en autorregulación por la Enfermería en Chile, son coincidentes con los antecedentes históricos y contextuales de la regulación establecidos por el Consejo Internacional de Enfermeras a nivel internacional $^{(22)}$. En la década de los ochenta esta organización internacional puso en marcha una iniciativa para fortalecer la reglamentación básica de la Enfermería en todo el mundo, encontrándose Chile entre los países latinoamericanos que han avanzado en su reglamentación ${ }^{(22)}$.

Finalmente, es pertinente hacer mención al análisis argumentativo a veinte años de gestión del cuidado en Chile de Ayala ${ }^{(23)}$ cuando señala: "el texto de la Ley de 1997 era ambiguo con respecto a las enfermeras, que prácticamente ignoraban la idea de la exclusividad ocupacional, lo que abrió el espacio para la jurisdicción compartida”. Esto obliga a replantearse la autorregulación de Enfermería, de modo de enfrentar los desafíos que emergen desde el interior y exterior de la profesión, como una fuerza laboral cambiante, la entrega de nuevos servicios de salud y nuevos problemas sociales que afectan a las enfermeras y la salud ${ }^{(23,24)}$. Lo anterior concuerda con lo señalado por Stahlke ${ }^{(25)}$ en relación al impacto que los procesos regulatorios tienen en la innovación de los roles de Enfermería.

\section{CONCLUSIÓN}

El avance que significó la introducción de la definición de Enfermería al Código Sanitario chileno, como proceso reivindicativo al mandato social de la labor de la enfermera, se constituyó en un hito político importante para el reconocimiento legal de la Enfermería como profesión pero que en la práctica continuó siendo subordinada a la estructura hegemónica al interior de las instituciones 
y profesiones de salud. Es decir, la validación y respaldo legal a la profesión se debe transformar en un empoderamiento del colectivo de enfermeras que propicie el cambio en la representación social de la profesión.

El respaldo legal a la función de la enfermera constituyó sin duda un avance importante para la profesión y su autonomía; a más de veinte años de este hito sociopolítico en que aparecen nuevos desafíos en el área de la salud y cuidado de las personas, cobra valor continuar con la autorregulación para consolidar su autonomía e identidad para defender con mayor convicción el sentido de ser de la Enfermería.

Finalmente se deja de manifiesto la necesidad de seguir avanzando en el posicionamiento a nivel nacional de la Enfermería en coherencia a los marcos regulatorios, que resguardan a la profesión y su quehacer, que a su vez deben validarla en el ámbito de la salud y en el ámbito social, de manera que la Enfermería nacional esté en consonancia con la identidad de la profesión a nivel internacional y con las directrices emanadas desde el Consejo Internacional de Enfermeras respecto a la autorregulación.

Las limitaciones de este estudio dicen relación con que los resultados presentados se basan en los testimonios de informantes como representantes de un grupo de interés del fenómeno social abordado en la investigación doctoral.

\section{REFERENCIAS}

1. Meleis A. Concept Development. En: Kluwer W, editor. Theoretical Nursing Development and progress. 5a Ed. Lippincott Williams \& Wilkins; 2012. p. 371-88.

2. Ten Hoeve Y, Jansen G, Roodbol P. The nursing profession: Public image, self-concept and professional identity. A discussion paper. J Adv Nurs. 2014; 70(2): 295-309.

3. Affara FA, Al-Jabri SS. Professional Self-Regulation for Nursing and Midwifery in Oman: Protecting the Public and Enhancing the Quality of Care. Oman Med J. 2016; 31(4): 243-4.

4. Benton DC, González-Jurado MA. BeneitMontesinos JV. Defining nurse regulation and regulatory body performance: a policy Delphi study. Int Nurs Rev. 2013; 60: 303-12.

5. Gitschel K, Ketteler V, Pfeiffer K. [The concept of professionalism in nursing: Between tradition and progress]. Pflege Z. 2006; 59(11): 2-7.
6. Jara P, Behn V, Ortiz N, Valenzuela S. Nursing in Chile. In: Breda KL, editor. Nursing and globalization in the Americas: a critical perspective. New York: Baywood Publishing Company; 2009. p. 53-97.

7. Decreto Ley 3621 fija Normas sobre Colegios Profesionales [Internet]. Valparaíso, Chile: Biblioteca Congreso Nacional; 1981 [citado $06 \mathrm{Abr}$ 2018]. Disponible en: https://www.leychile.cl/ $\mathrm{N} ? \mathrm{i}=7195 \& \mathrm{f}=1981-02-07 \& \mathrm{p}=$

8. Código Sanitario (DFL 725/1967). El artículo 113 fue modificado por el artículo $7^{\circ}$, letra a) de la ley № 19.536 que incorpora la definición de los servicios profesionales de la enfermera en su inciso cuarto [Internet]. Valparaíso, Chile: Biblioteca Congreso Nacional; 1997 [citado 06 Abr 2018]. Disponible en: https://www.leychile.cl/ Navegar?idNorma $=5595 \#$ enfermera0

9. Duncan S, Thorne S, Rodney P. Evolving trends in nurse regulation: what are the policy impacts for nursing's social mandate? Nurs Inq. 2015; 22(1): 27-38.

10. Crotty M. The Foundations of Social Research: Meaning and Perspective in the Research Process. London: SAGE Publications Ltd; 1998.

11. Chinn PL, Kramer MK. Emancipatory Knowledge Development. In: Chin PL, Kramer MK, editors. Integrated Theory and Knowledge Development in Nursing. 8th Ed. St. Luis, Missouri: Mosby Elsiever; 2011. p. 63-86.

12. Kagan PN, Smith MC, Chinn PL, editors. Philosophies and practices of emancipatory nursing: Social justice as praxis. New York: Routledge; 2014. 360 p.

13. Nejatian A, Joulaei H. Contextual factors for establishing nursing regulation in Iran: A Qualitative Content Analysis. Int J Community Based Nurs Midwifery. 2018; 6(2): 125-35.

14. Milos Hurtado P, Bórquez Polloni B, Larrain Sundt AI. La "gestión del cuidado"; en la legislación chilena (II) estado actual. Cienc Enferm. 2011; 17(3): 23-33.

15. National Council of State Boards of Nursing. The Nursing Regulatory Environment in 2018: Issues and Challenges. J Nurs Regul. 2018; 9(1): 52-67.

16. Tanaka M, Taketomi K, Yonemitsu Y, Kawamoto R. Professional behaviours and factors contributing to nursing professionalism among nurse managers. J Nurs Manag 2016; 24(1): 12-20.

17. Morgan J, Hopkins W, Acreman S, Jewell K, Garwood L, Candy E. What does professionalism look like? Attitudes and behaviours derived from a Delphi study. Nurs Manag. 2014; 21(7): 28-40.

18. Pavez Lizarraga A. La enfermería, realidad de ciudadanía y de género en Chile. Cienc enferm. 2013; 19(3): 95-102. 
19. Fenwick T. Professional responsibility and professionalism: A sociomaterial examination. New York: Routledge; 2016. 228 p.

20. Stievano A, Caruso R, Pittella F, Shaffer FA, Rocco G, Fairman J. Shaping nursing profession regulation through history-as sistematic review. Int Nurs Rev. 2018; 66(1): 17-29.

21. Wynd CA. Current factors contributing to professionalism in nursing. J Prof Nurs. 2003; 19(5): 251-61.

22. Heale R, Rieck Buckley C. An international perspective of advanced practice nursing regulation. Int Nurs Rev. 2015; 62(3): 421-9.

23. Ayala RA. Twenty years of management of care in Chile: what we know, what we do not know, what is yet to come. An analysis of arguments. Med Humanit [Internet]. 2018 [cited 2018 Jul 16]; 0: 1-11 Available from: https://mh.bmj.com/ content/early/2018/07/15/medhum-2017-011394. long

24. Siles JG. Epistemología y enfermería: por una fundamentación científica y profesional de la disciplina. Enferm Clin. 1997; 7(4): 188-94.

25. Stahlke Wall S. The impact of regulatory perspectives and practices on professional innovation in nursing. Nursing Inquiry [Internet]. 2018 Jan [cited 2019 May 1]; 25(1): 1. Available from: http://bibliotecadigital.umayor.cl:2088/login.asp $\mathrm{x}$ ?direct=true $\& \mathrm{db}=\mathrm{ccm} \& A N=127390068 \&$ lang= es\&site $=$ ehost-live 